\title{
Une étude exploratoire du procédé d'hydroformage des tôles
}

\section{An Exploratory Study of the Sheet Hydroforming Process}

\author{
Mohammed Nassraoui ${ }^{1}$, Bouchaib Radi ${ }^{2}$ \\ ${ }^{1}$ Laboratoire LMPGEI, Ecole Supérieure de Technologie - Université Hassan II, Casablanca, Maroc, nassraoui@yahoo.fr \\ ${ }^{2}$ Laboratoire LIMII, Faculté des Sciences et Techniques - Université Hassan I, Settat, Maroc, bouchaib.radi@yahoo.fr
}

RÉSUMÉ. Cet article présente une étude exploratoire du procédé d'hydroformage d'une tôle. Avant de procéder aux simulations, on a réalisé un essai expérimental pour valider le modèle mathématique de simulation par éléments finis. Un contrôle géométrique expérimental permet de déterminer l'allure de la déformée afin de la comparer avec les allures de simulation. Les résultats de simulation ont permis de choisir les paramètres nécessaires pour avoir une bonne déformée. ABSTRACT. This article presents an exploratory study of the hydroforming process of a sheet. Before proceeding to the simulations, an experimental test was carried out to validate the mathematical model of simulation by finite elements. An experimental geometrical control makes it possible to determine the shape of the deformation in order to compare it with the simulation looks. The simulation results made it possible to choose the parameters necessary to have a good deformation.

MOTS-CLÉS. Hydroformage, contrôle géométrique, éléments finis, allure de déformée.

KEYWORDS. Hydroforming, geometric control, finite elements, distorted appearance.

\section{Introduction}

L'hydroformage est un procédé de formage utilisant un fluide actif (souvent une émulsion eauhuile). Une pièce creuse est formée à partir d'un tube, d'un profilé ou de deux flans, par l'application d'une pression interne. La pression nécessaire dans ce process dépend énormément du matériau utilisé, de l'épaisseur du matériau et du plus petit rayon qui doit être formé. La pression interne nécessaire peut monter jusqu'à plusieurs milliers de bars [Yua06].

Les avantages de ce process de formage par rapport à l'emboutissage profond classique sont les suivants :

- Conception pour des pièces complexes.

- Minimisation des jonctions entre les pièces assemblées et/ou soudées.

- Réduction du matériau utilisé et du poids de la pièce.

- Durabilité plus longue.

- Précision dimensionnelle et précision de formage avec un retour élastique réduit.

Les avantages de ce procédé de formage est trop demandé dans l'industrie automobile comme d'autres secteurs industriels. Par exemple, l'industrie de la plomberie et du chauffage, la technologie de l'électro-ménager, l'aviation et l'aérospatiale, l'industrie du mobilier ainsi que la construction de machines et d'équipements [OHIO6].

Dans l'industrie automobile, l'hydroformage est essentiellement utilisé pour fabriquer des composants de systèmes d'échappement et divers châssis et pièces de carrosserie. Par exemple, des moteurs, des traverses et longerons, des structures de toit mais aussi des pieds avant, pieds milieu et pieds arrière $[$ Kah10] . 

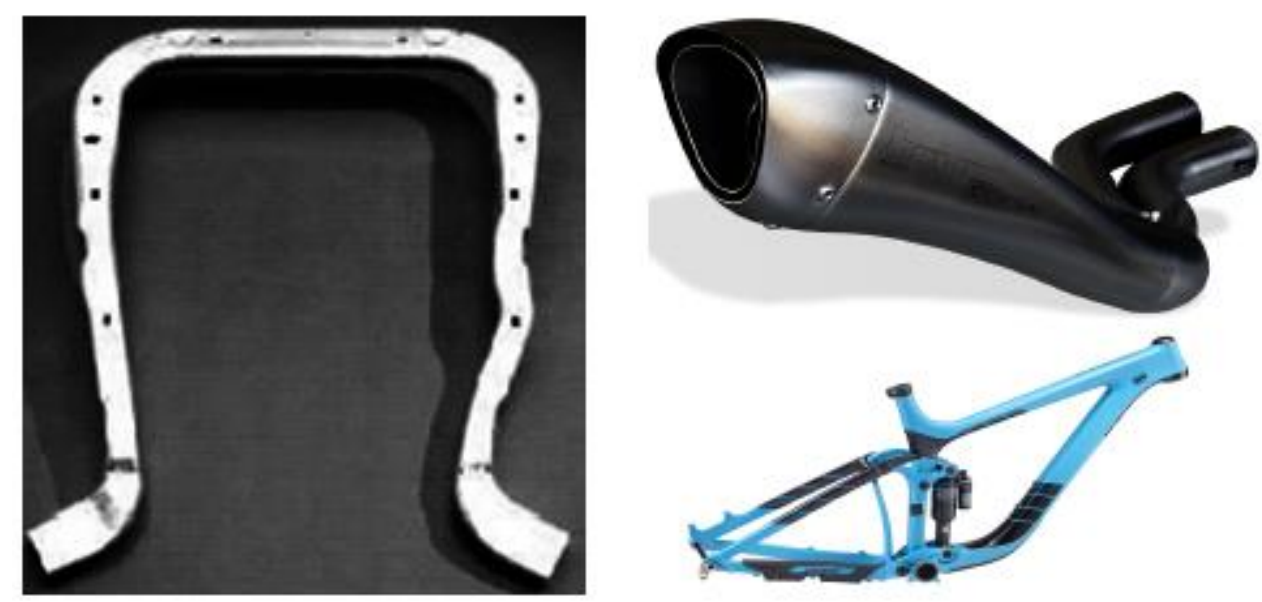

Figure 1. Exemple de pièces hydroformées - Source : Internet

\section{Principe du procédé}

L'hydroformage est un procédé de fabrication par déformation. Il consiste à déformer plastiquement des tôles ou des tubes dans une matrice en utilisant une pression hydraulique contrôlée. La principale caractéristique de ce procédé est que le poinçon est remplacé par l'action directe de la pression du liquide (eau, huile hydraulique, émulsion) [Hei99]. Le cycle typique comprend l'insertion de la pièce métallique à l'intérieur d'une estampe ou d'un moule, la fermeture du moule et l'application de la pression du liquide à l'intérieur du tube ou d'un seul côté de la tôle (voir figure 2).
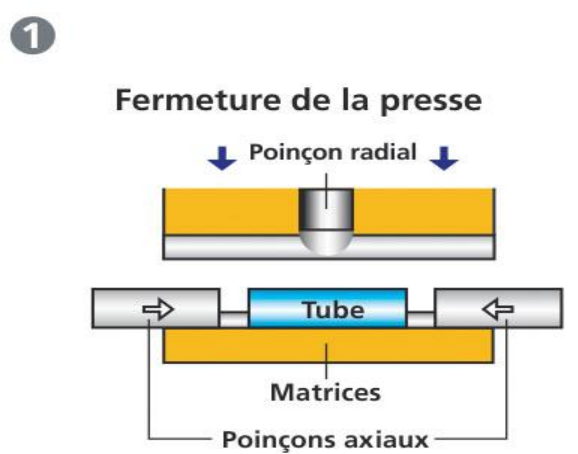

3
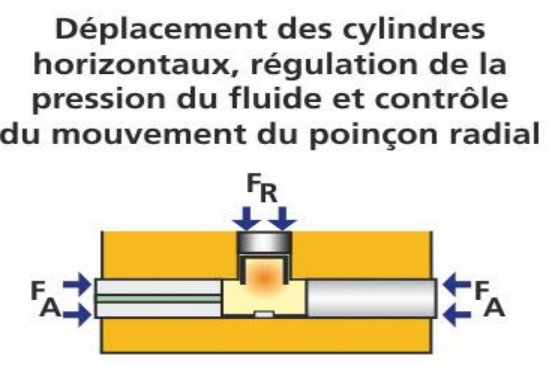

2

Remplissage avec le fluide

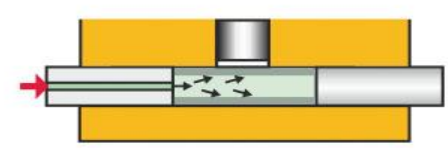

$(4)$

Ouverture de la presse, extraction de la pièce

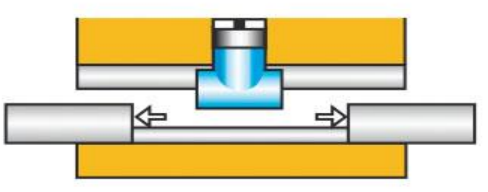

Figure 2. Principe du procédé d'hydoformage - Source : Société Autoform

Plusieurs techniques du procédé d'hydroformage ont été développées. Certaines de ces techniques du même procédé sont extrêmement différentes. Certaines utilisent une matrice femelle et d'autres une matrice mâle, certaines n'utilisent pas de liquide mais plutôt un matériau élastomère alors que d'autres utilisent un liquide directement sur le matériau à former [LAN 14]. 


\section{Expérimentation}

\subsection{Essai d'hydroformage}

L'essai a été effectué avec le matériel illustré dans la figure 3. Le test a consisté à emboutir une tôle circulaire de diamètre $205 \mathrm{~mm}$ et d'une épaisseur de $2 \mathrm{~mm}$ en acier S235. La pression de formage varie jusqu'à une pression de $2 \mathrm{MPa}$ tout en utilisant la méthode de serrage de la tôle. La matrice utilisée lors des essais est une matrice circulaire, d'un diamètre intérieur de $198 \mathrm{~mm}$. et d'un rayon de matrice de 2 $\mathrm{mm}[\mathrm{Nas} 18]$.
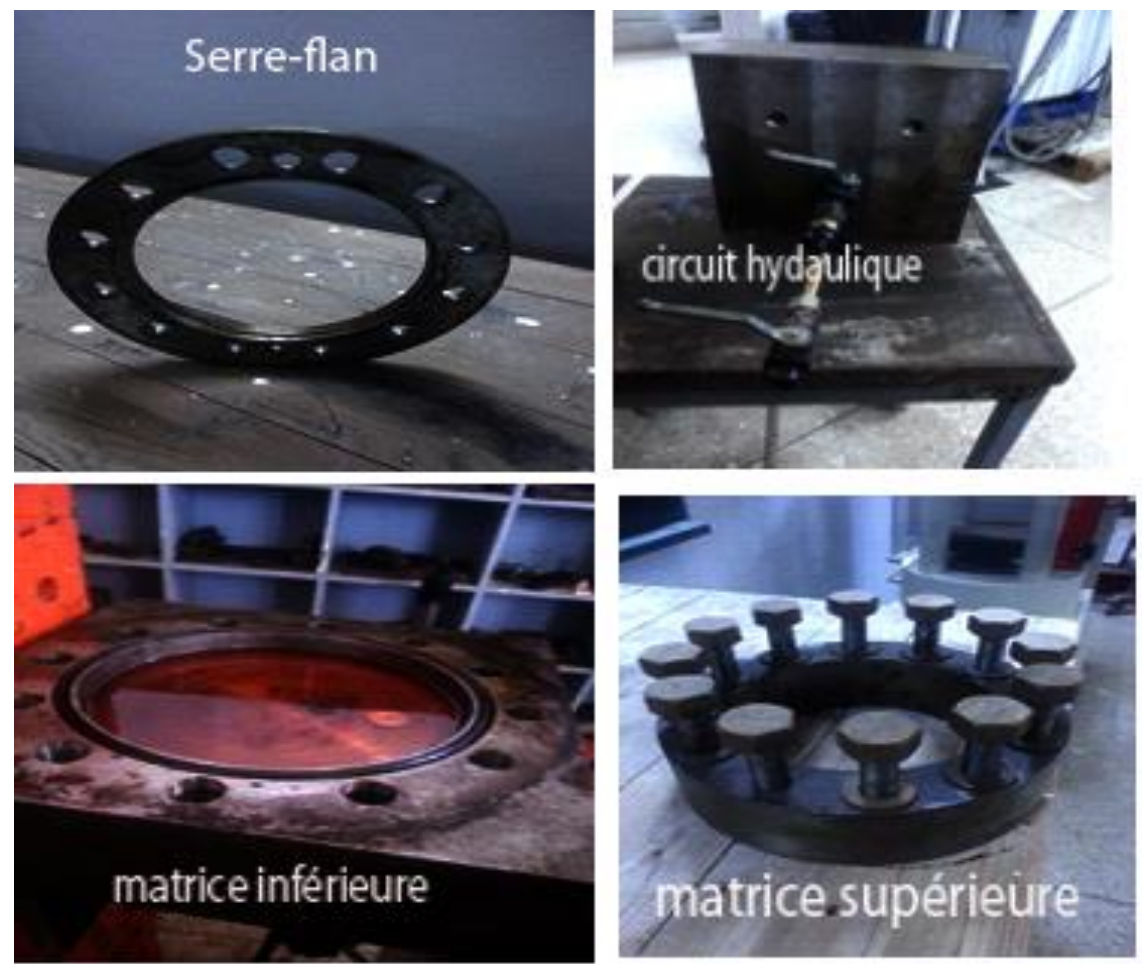

Figure 3. Composants du banc d'essai - Source: Laboratoire ENSEM

\subsubsection{Description de l'essai}

Après avoir effectuer tous les réglages de vérification d'étanchéité et d'étalonnage des différents capteurs d'acquisition de déplacement et de pression (voir figure 4).

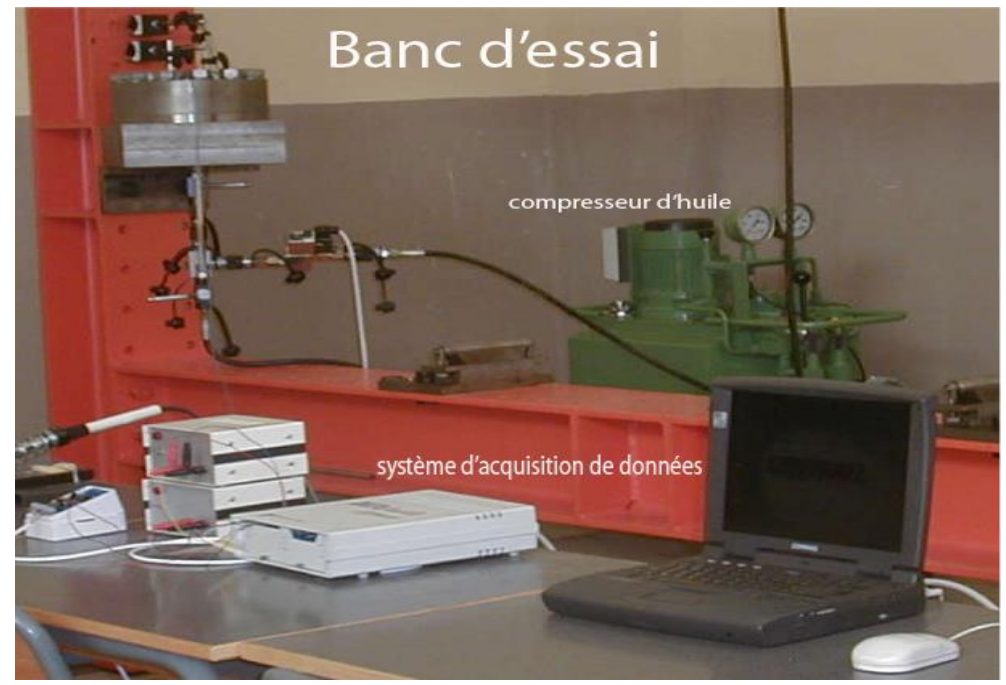

Figure 4: Système d'hydroformage - Source: Laboratoire ENSEM

On a procédé à la déformation de la tôle concernée (voir figure 5). 

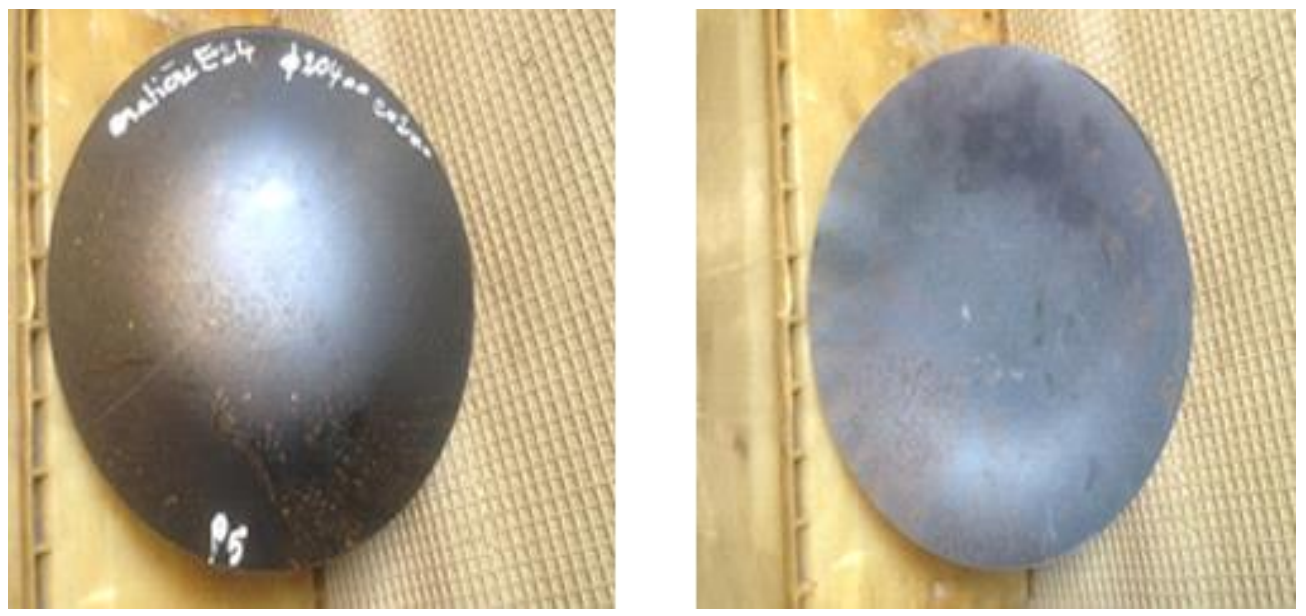

Figure 5. Tôle hydroformée- Source: Laboratoire ENSEM

\subsubsection{Résultats de l'essai}

La courbe suivante (figure 6) illustre la variation de la déformée du centre de la tôle en fonction de la pression alimentée. Pour une pression de 20 bar $(2 \mathrm{MPa})$, le déplacement au centre de la tôle atteint une valeur de $10,9 \mathrm{~mm}$.

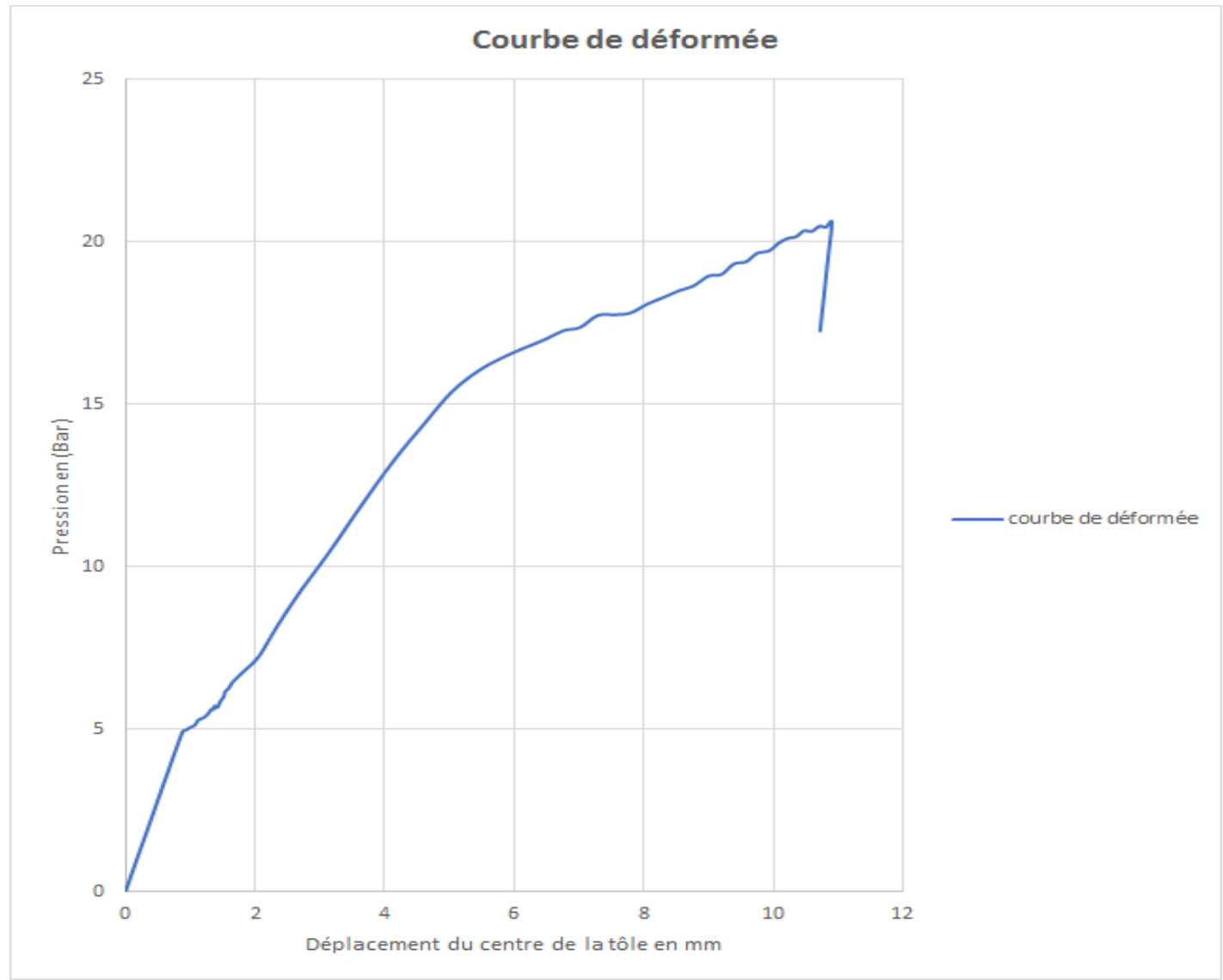

Figure 6. Courbe de la déformée

\subsection{Mesure de l'allure de la déformée}

Afin d'analyser la géométrie de la tôle hydroformée, on utilisera une machine à mesurer tridimensionnelle (MMT) pour comparer la forme mesurée avec celle qui sera simulée. 


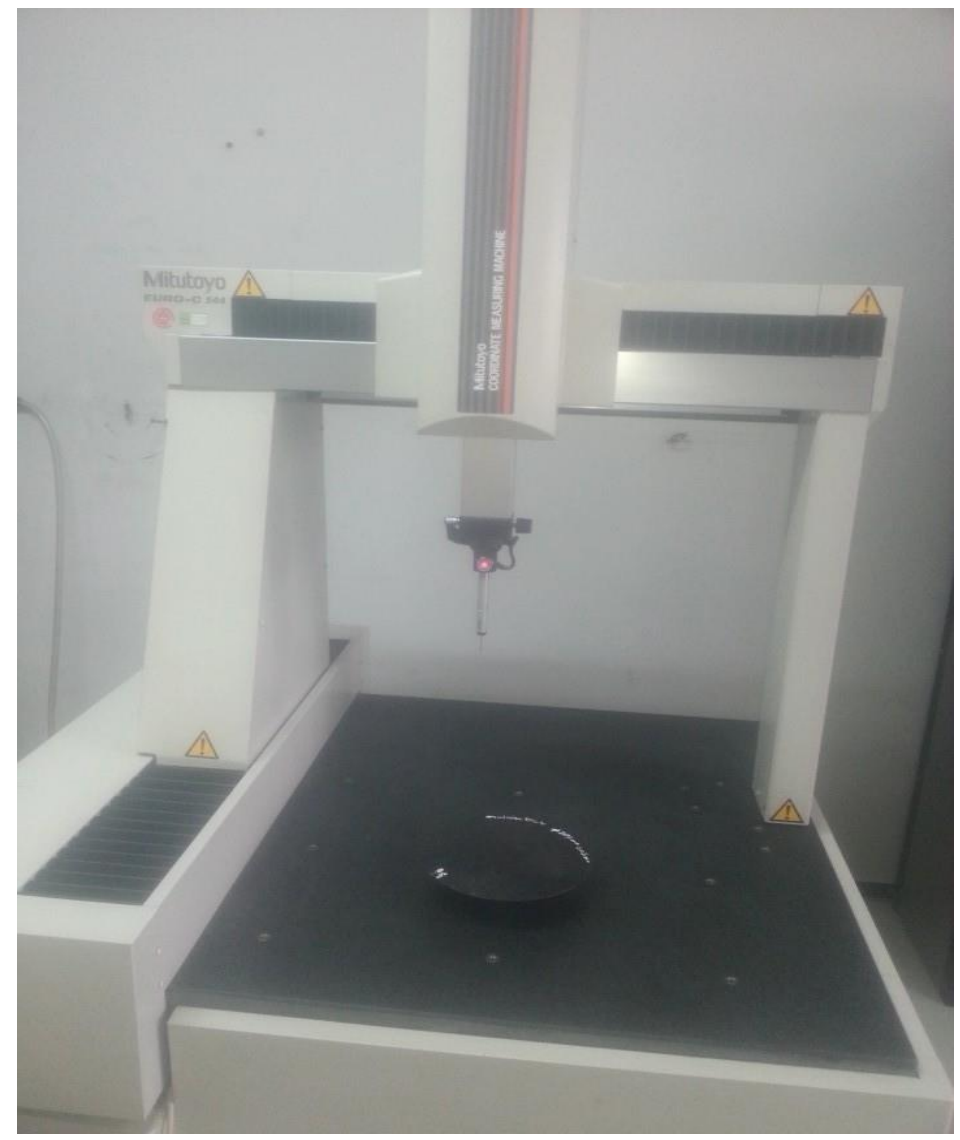

Figure 7. Mesure de la tôle sur MMT: Labo ENSET-Rabat

La courbe 8 montre l'allure de la courbe de la tôle hydroformée.

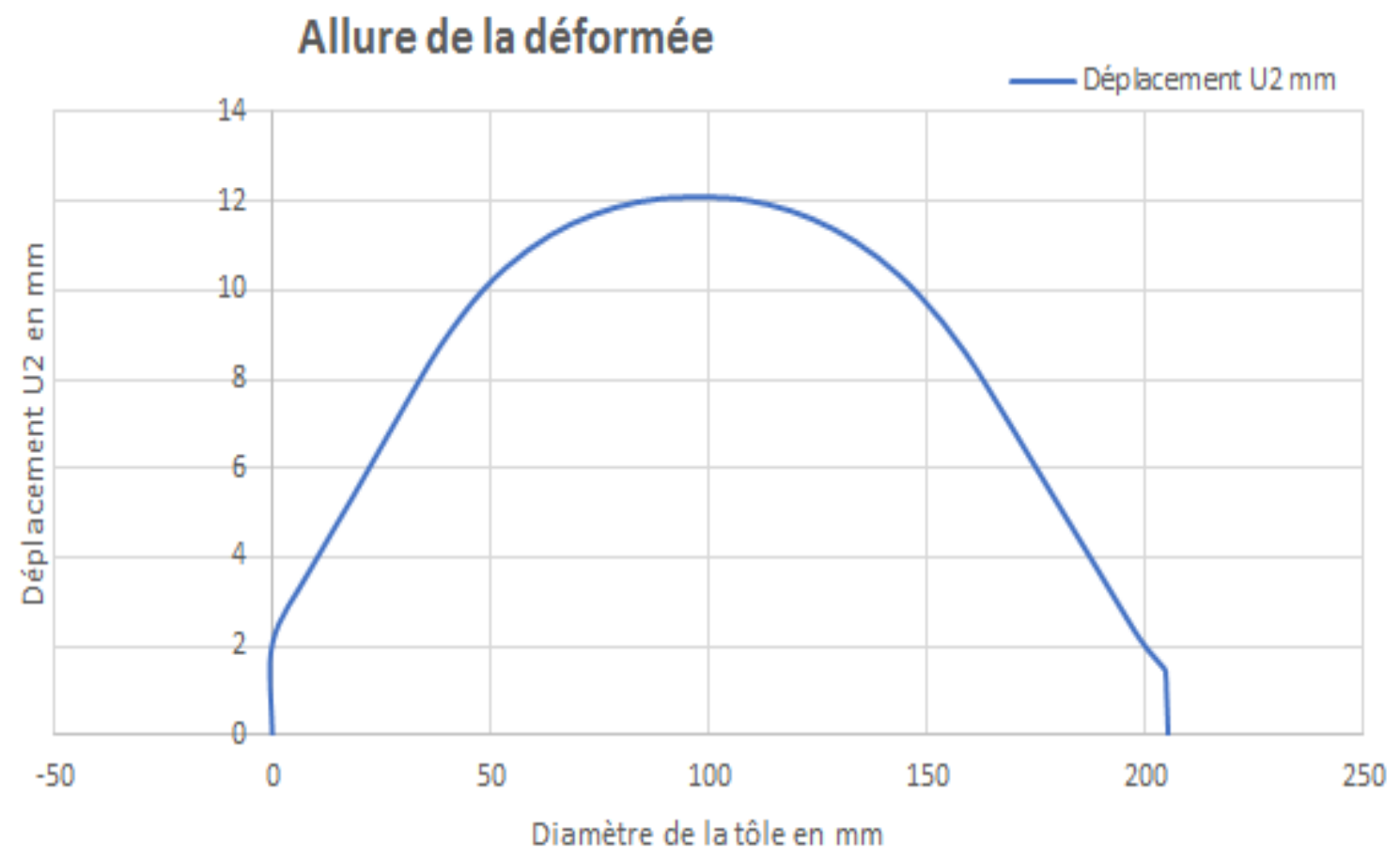

Figure 8. Allure expérimentale de déplacement 


\section{Simulation numérique}

Pour vérifier la formabilité de la tôle par le procédé d'hydroformage, nous avons pris un modèle de simulation par éléments finis à l'aide du logiciel Abaqus. Ce logiciel a été utilisé pour tenir compte des non linéarités de l'hydroformage et du contact entre la tôle et la matrice.

\subsection{Modélisation de l'essai}

Nous avons modélisé la matrice et la tôle sur le module «PART» du logiciel en tenant compte des dimensions réelles.

La tôle a été représentée en mode surfacique mais la matrice en mode solide. Avant de procéder à l'assemblage, des propriétés de matériau sont associées aux pièces. Le matériau de la tôle a été créé puisqu'elle est la seule qui se déforme.

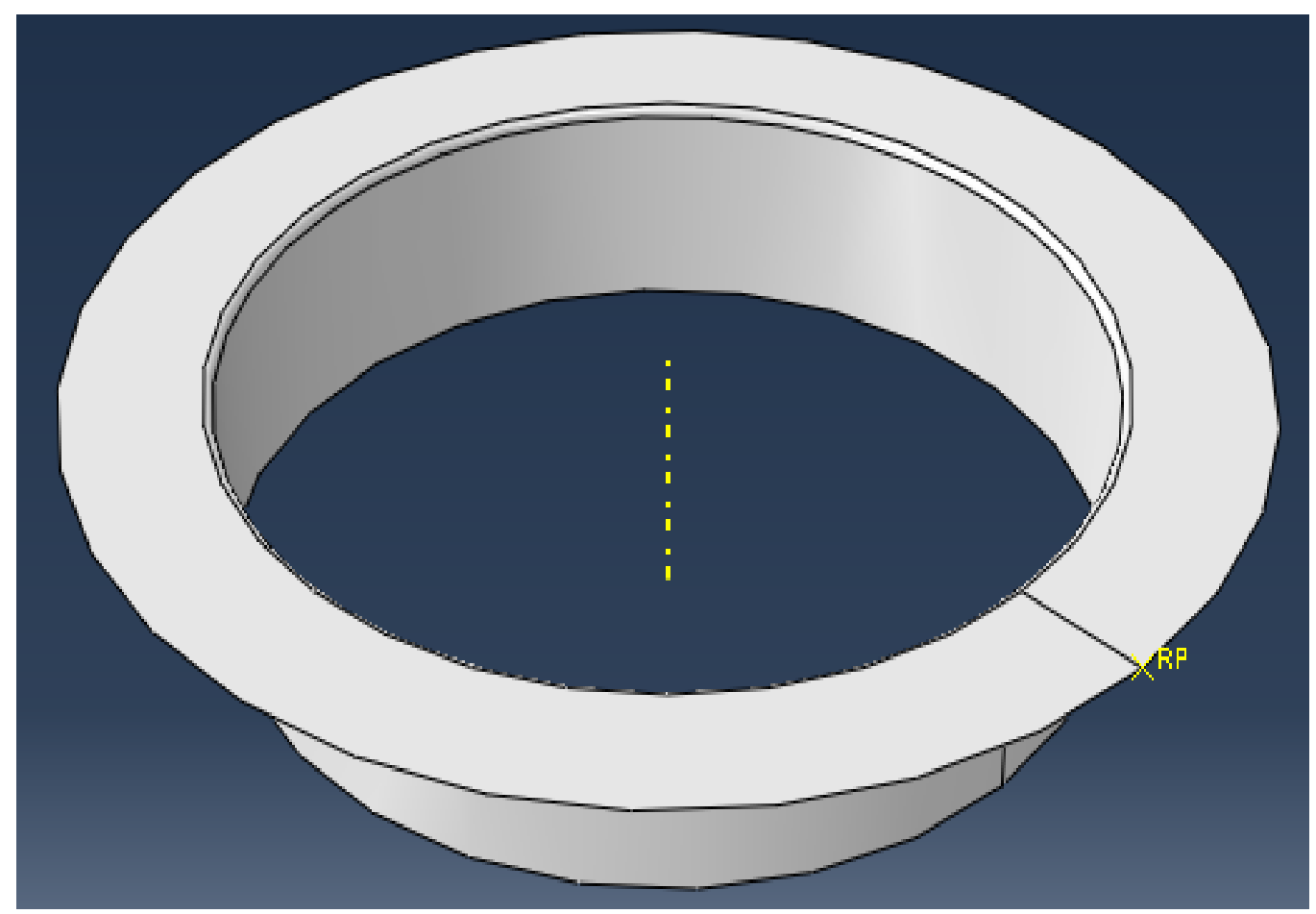

Figure 9. Matrice

Les différentes propriétés du matériau (S235) sont la densité, le module de Young, le cœefficient de Poisson et les données reliées au domaine plastique.

Pour analyser le domaine plastique, la méthode élastoplastique est utilisée car la tôle subit des déformations élastiques avant de se déformer de façon plastique.

- Module de Young : $210000 \mathrm{MPa}$

- Coefficient de Poisson : 0,3

- Densité : $7850 \mathrm{Kg} / \mathrm{m} 3$

- contraintes plastiques: (voir Tableau 1 ) 


\begin{tabular}{|c|c|c|c|c|c|}
\hline $\begin{array}{c}\text { Contrainte } \\
(\mathbf{M P a})\end{array}$ & $\begin{array}{c}\text { Déformations } \\
\text { plastiques }\end{array}$ & $\begin{array}{c}\text { Contrainte } \\
(\mathbf{M P a})\end{array}$ & $\begin{array}{c}\text { Déformations } \\
\text { plastiques }\end{array}$ & $\begin{array}{c}\text { Contrainte } \\
(\mathbf{M P a})\end{array}$ & $\begin{array}{c}\text { Déformations } \\
\text { plastiques }\end{array}$ \\
\hline 235 & 0 & 272.42 & 0.01115882 & 329.53 & 0.07856373 \\
\hline 238.15 & 0.0034437 & 276.23 & 0.01277138 & 333.34 & 0.08874996 \\
\hline 241.96 & 0.00389327 & 280.03 & 0.01461445 & 337.15 & 0.1001401 \\
\hline 245.76 & 0.00441476 & 283.84 & 0.01671752 & 340.96 & 0.11286063 \\
\hline 249.57 & 0.00501873 & 287.65 & 0.01911339 & 344.76 & 0.12704967 \\
\hline 253.38 & 0.00571714 & 291.46 & 0.02183846 & 348.57 & 0.14285791 \\
\hline 257.19 & 0.00652344 & 295.269 & 0.02493311 & 352.38 & 0.16044958 \\
\hline
\end{tabular}

Tableau 1. Valeur de contraintes plastiques

Une section est associée à la tôle déformable de type coque. L'épaisseur de la tôle $2 \mathrm{~mm}$ est définie dans cette section pour aborder le mode d'intégration. Pour évaluer le comportement du matériau à chaque point d'intégration, on choisit la méthode de Gauss pour raison de précision et réduction du temps de calcul.

Pour faciliter la déformation de la tôle dans la matrice pendant que la pression augmente, plusieurs étapes sont créées afin de modéliser l'essai réel. Une pression ou une charge « suiveuse » variable urant le temps sur la surface d'application d'une valeur initiale de $0 \mathrm{MPa}$ à une valeur finale de $2 \mathrm{MPa}$.

Le modèle est crée sous un état quasi-statique avec un état initial de la matrice et la tôle. Lors de la simulation statique d'hydroformage, le facteur frottement entre les surfaces de la matrice et la tôle flan a une très grande influence sur le résultat du formage.

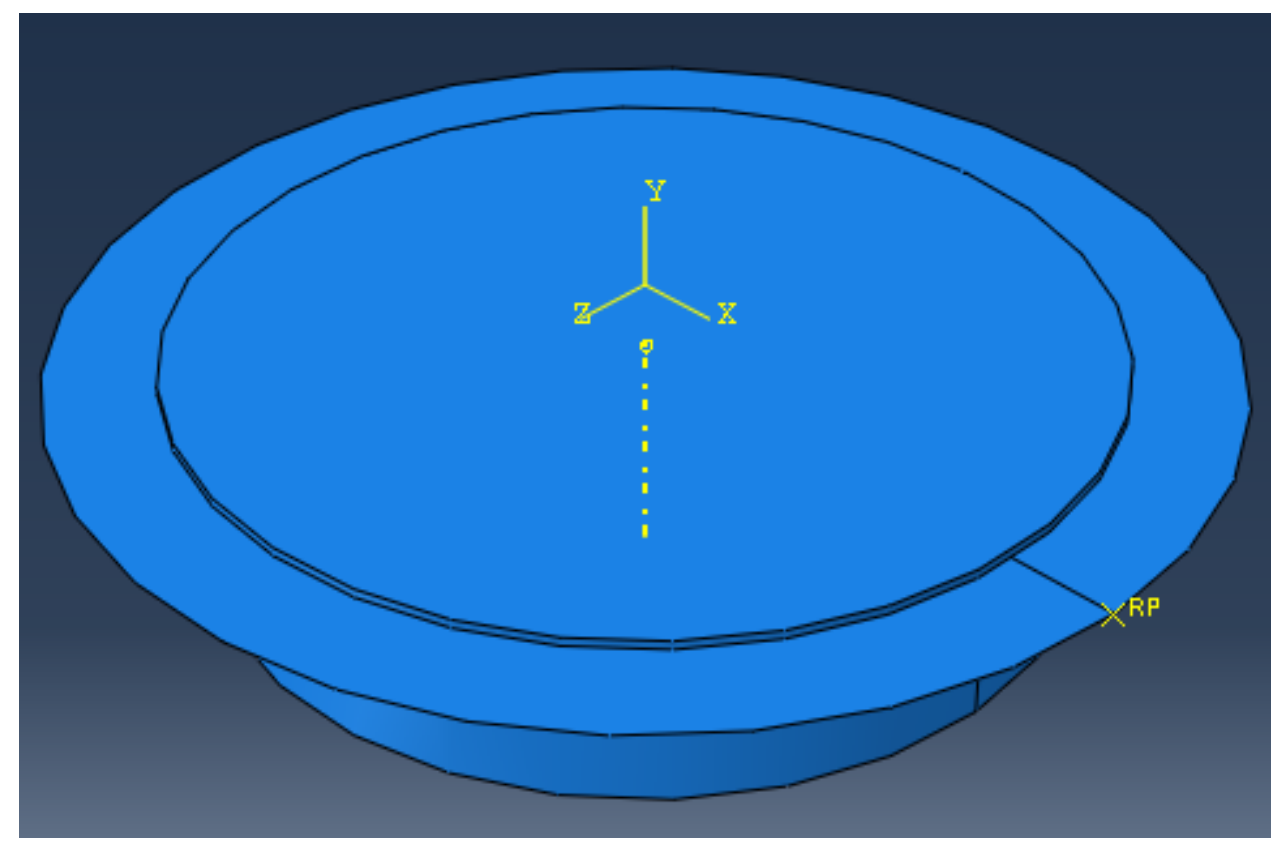

Figure 10. Assemblage tôle matrice

Les forces de friction influencent sur le bon déroulent du process d'hydroformage, particulièrement sur la formation de froissement, la localisation des variations d'épaisseur et les défauts des déchirures [BOU 20]. Dans notre cas on a réalisé deux simulations la première avec un coefficient de frottement $\mu$ de 0,5 et la deuxième sans frottement. 
La méthode de Coulomb entre deux surfaces en contact peuvent accepter un certain degré de contraintes en cisaillement avant de commencer à glisser l'une par rapport à l'autre [LAM 10]. Le modèle de Coulomb définit une contrainte de cisaillement critique . La contrainte critique est calculée avec l'équation suivante :

$$
\tau_{\text {Critique }}=\mu P \text {. }
$$

où $\mathrm{P}$ est la pression de contact.

Le modèle de friction suppose que $\mu$ est le même dans toutes les directions. Il y a deux composantes de friction orthogonales $\tau_{1}$ et $\tau_{2}$. ABAQUS suppose les deux composantes exercées sur les deux surfaces en contact comme une contrainte de cisaillement équivalente:

$$
\bar{\tau}=\sqrt{\tau_{1}{ }^{2}+\tau_{2}{ }^{2}} .
$$

Afin vérifier l'influence du maillage, nous avons réalisés plusieurs simulations avec différents types (grossier, fin et très fin). Nous avons constaté que le type de maillage de la tôle n'a pas d'effet sur les résultats des simulations.

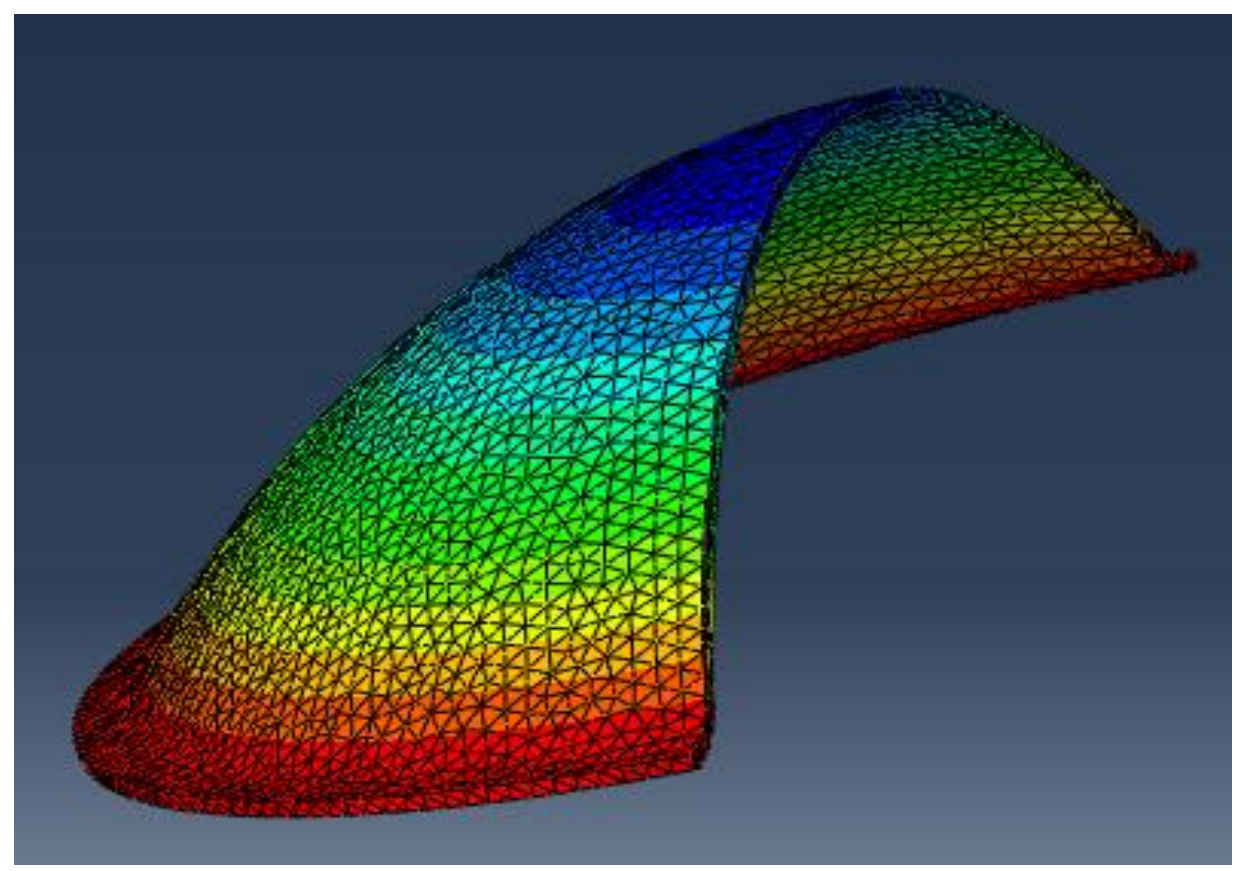

Figure 11. Tôle déformée

\subsection{Résultats de simulation}

Après réalisation de plusieurs simulations numériques, on a constaté que la déformée dépend de beaucoup de paramètres tels que :

- La force de serrage au niveau du bord de la tôle.

- La surface de contact et le rayon de courbure de la matrice ce qui permet un bon écoulement de la matière.

- Le cœfficient de frottement entre la matrice et la tôle.

Les figures 12 et 13 montrent la valeur de la déformée :

- Pour la tôle simulée sans frottement, la valeur de U2 max est de $16,98 \mathrm{~mm}$ 
- Pour la tôle simulée avec un coefficient de frottement de valeur $(\mu=0.5)$, la valeur de U2 max de $8,868 \mathrm{~mm}$

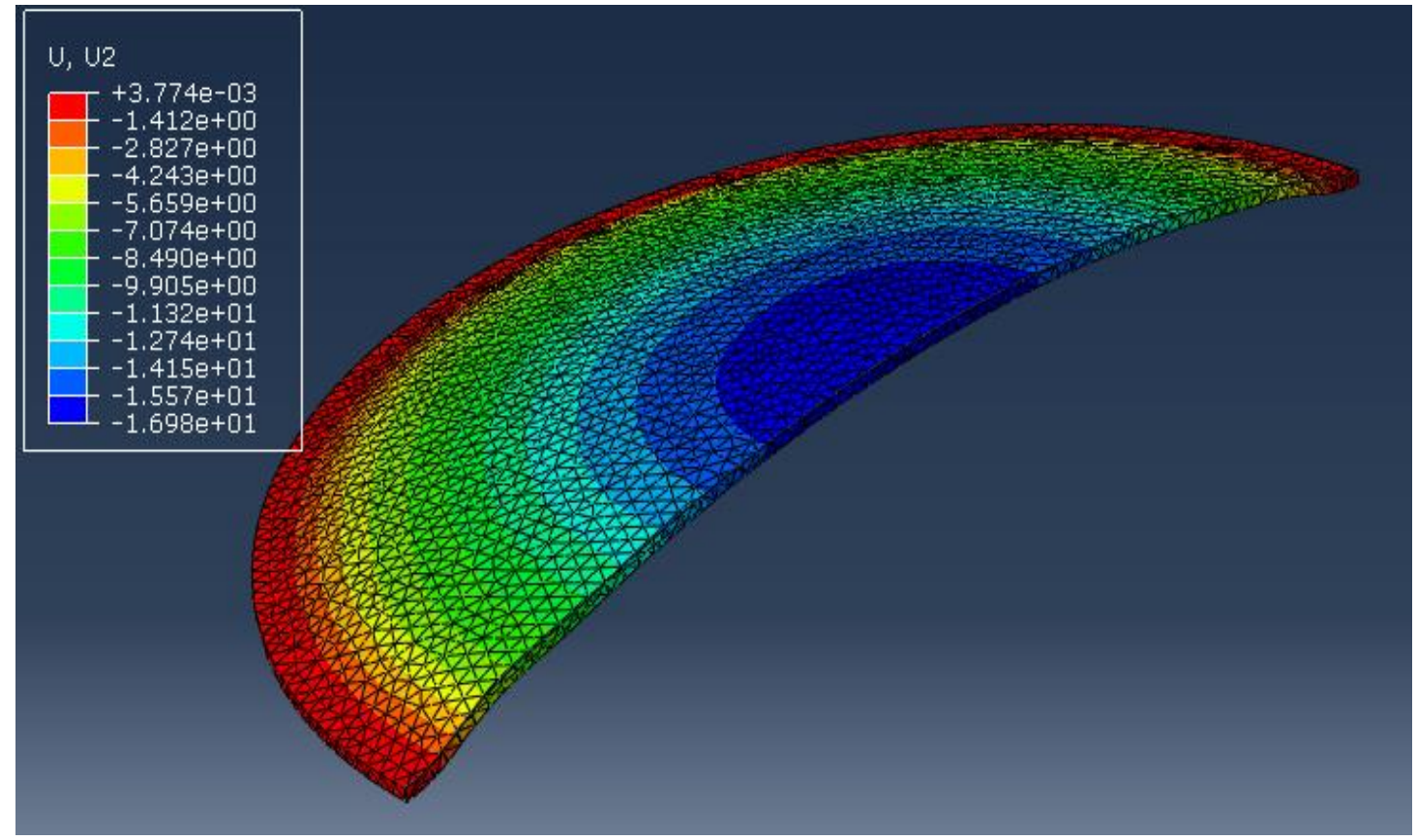

Figure 12. Tôle hydroformée sans frottement

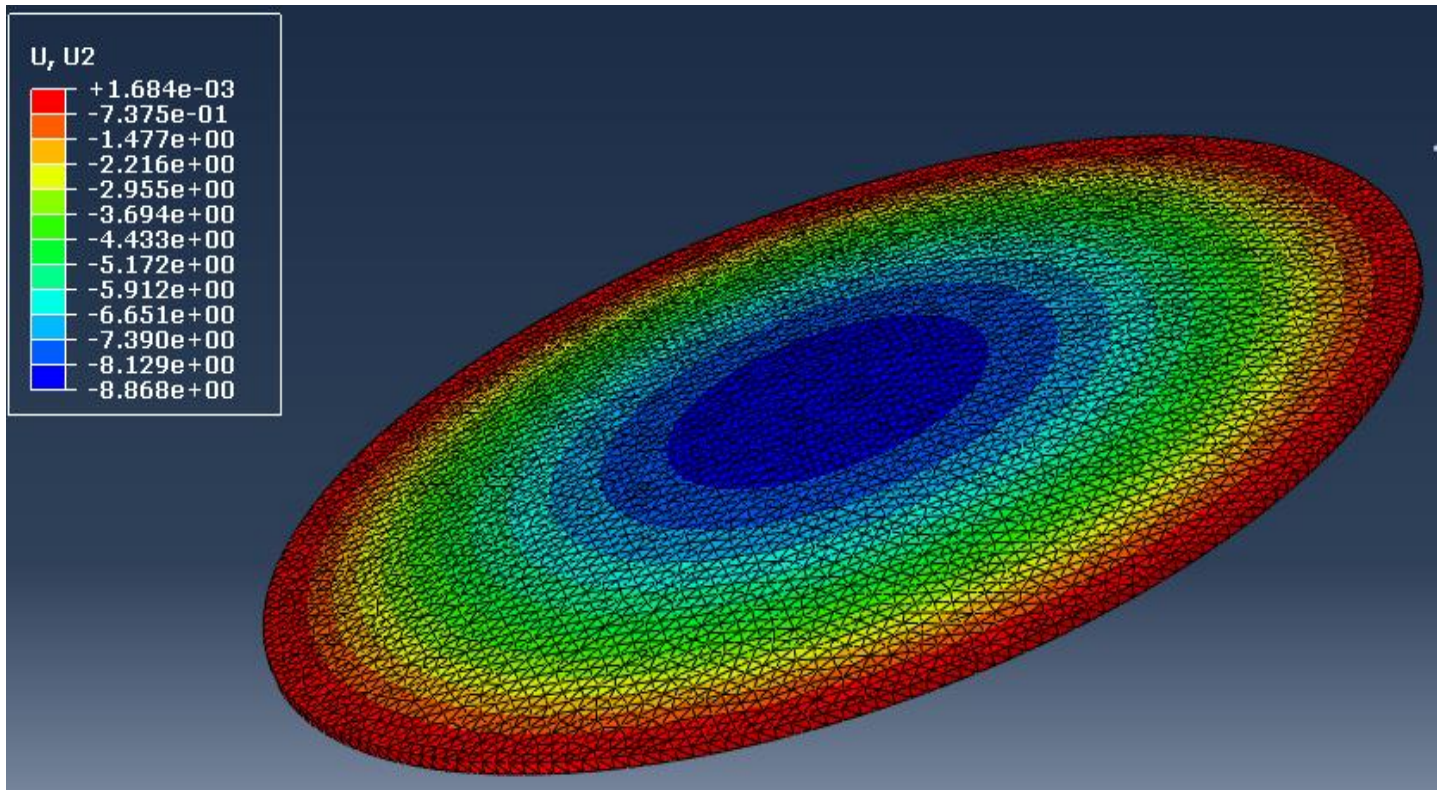

Figure 13. Tôle hydroformée avec frottement

La comparaison de la déformée obtenue par simulation, en considération du frottement ou sans frottement, avec la déformée expérimentale permet de valider le modèle défini dans ABAQUS. La courbe figure 14 permet de visualiser les allures de simulation et expérimentale. 


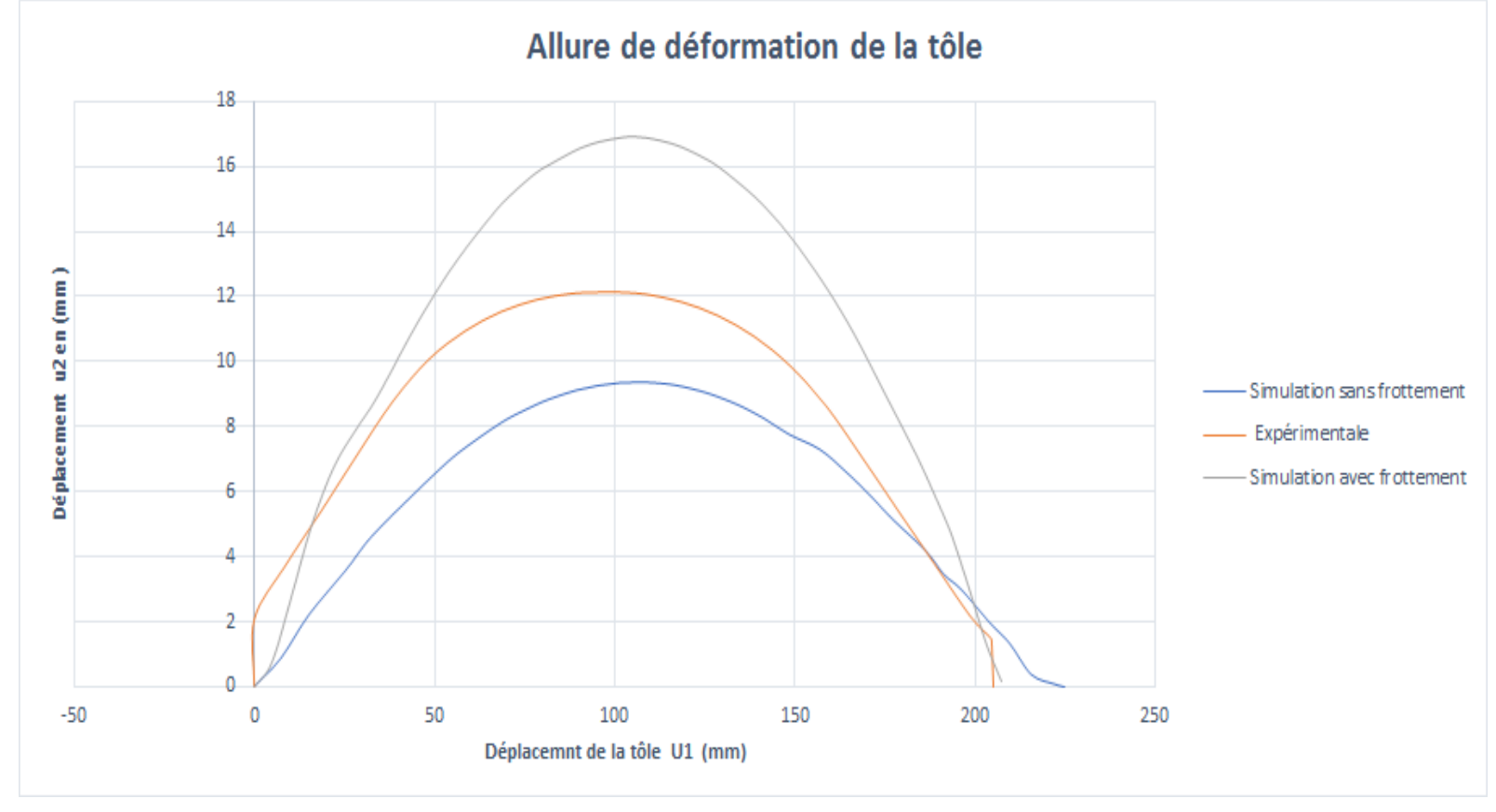

Figure 14. Allure de déformation de la tôle

\section{Conclusion}

La comparaison des allures montre que la simulation donne un résultat assez semblable a celui du formage réel. Le modèle de frottement et les propriétés du matériau sont valables pour la simulation d'un hydroformage par action directe du fluide sur la tôle. L'utilisation d'un liquide de pression qui a une bonne viscosité permet de réduire la force de friction aux interfaces ce qui facilite l'écoulement de métal dans la matrice.

\section{Bibliographie}

[BOU 20] Boulbes R.J., « Troubleshooting Finite-Element Modeling with Abaqus», Springer, Cham, France, 2020.

[LAN 14] LANG L.H., WANGZ R., KANG D.C., «Hydroforming highlights: sheet hydroforming and tube hydroforming.», Journal of Materials Processing Technology , $\mathrm{N}^{\circ}$ 1: p. 165-177, 2014.

[LAM 10] LAMONTAGNE L., «Etude sur l'application de l'hydroformage » mémoire, Université Laval - Quebec, 2010.

[Hei99] HeIN P. and VolLERTSEN F., «Hydroforming of sheet metal pairs », Journal of Materials Processing Technology, 87: 154-164, 1999.

[Kah10] KAHOUL H., «Modélisation du comportement à la déformation élastique des tôles de construction automobile : influence des singularités », Master's thesis, Université Mentouri, Constantine - Algérie, 2010.

[OHI06] OH S.I., JEON B. H., KIM H. Y., and YANG J. B., « Applications of hydroforming processes to automobile parts », Journal of Materials Processing Technology, 174: 42-55, 2006.

[Yua06] YUAN S. J., HAN C. and WANG X. S., «Hydroforming of automotive structural components with rectangularsections », International Journal of Machine Tools and Manufacture, 46: 1201-1206, 2006.

[Nas18] NASSRAOUI M., ELMASKAOUI Z. and RADI B., «Experimental characterization of hydroforming», Incertitudes et fiabilité des systems multiphysiques (ISTE), No.2, May 2018. 